\title{
Síntomas gastrointestinales en pacientes receptores de trasplante renal de un centro en México
}

\author{
Gastrointestinal symptoms in kidney transplant \\ recipients from a center in Mexico
}

\author{
Yamilli Rivera-Sánchez,* Mónica Martínez-Maldonado,* \\ Luis Roberto Reyes-Constantino,* Ángela Domínguez-Rodríguez,* \\ Félix Guillermo Márquez-Celedonio, ${ }^{\ddagger}$ Luis Filadelfo Budar-Fernández, ${ }^{\S}$ \\ Pedro Iván Moreno-Ley, ${ }^{\S}$ Andrea Nachón-Acosta, ${ }^{\circledR}$ Gustavo Martínez-Mier ${ }^{\S}$ \\ * Escuela de Medicina, Universidad del Valle de México, Universidad Villa Rica, Boca del Río, Veracruz, México. \\ ‡ Departamento de Investigación, Universidad del Valle de México, Universidad Villa Rica, Boca del Río, Veracruz, México. \\ $\S$ Departamento de Trasplantes, IMSS, UMAE HE14 «Adolfo Ruiz Cortines», Veracruz, México. \\ " Departamento de Cirugía IMSS, UMAE HE14 «Adolfo Ruiz Cortines», Veracruz, México.
}

\section{RESUMEN}

Introducción: Los síntomas gastrointestinales son uno de los efectos posteriores al trasplante y parecen relacionarse a múltiples factores. El objetivo del estudio fue determinar la prevalencia de síntomas gastrointestinales en una cohorte mexicana de receptores de trasplante renal y su probable asociación al régimen inmunosupresor. Material y métodos: Se encuestó a pacientes receptores de trasplante renal mayores de 18 años, con función renal y tratamiento inmunosupresor de febrero a mayo de 2019. Los síntomas gastrointestinales fueron evaluados mediante el cuestionario Gastrointestinal Symptom Rating Scale (GSRS). Las puntuaciones totales más altas indican mayor severidad en los síntomas. Se evaluaron las diferencias en la puntuación GSRS tanto total como por síntoma específico. Se consideró una $\mathrm{p}<0.05$ como estadísticamente significativa. Resultados: Se encuestaron 103 pacientes (masculinos, $63.15 \%$ ), edad promedio 36.6 años, tiempo promedio postrasplante 45.5 meses. De los pacientes, 92 (91\%) tuvieron un síntoma gastrointestinal. Los síntomas más comunes fueron dolor de apetito $(65 \%)$, eructos $(49.5 \%)$ y heces sueltas (42.7\%). Los pacientes femeninos tuvieron mayor puntuación GSRS que los masculinos ( $\mathrm{p}<0.05$ ). La hemoglobina tuvo una

\section{ABSTRACT}

Introduction: Gastrointestinal symptoms are post-transplant side effects that appear to be related to multiple factors. The objective of the study was to determine the prevalence of gastrointestinal symptoms in a Mexican cohort of renal transplant recipients and their possible association with the immunosuppressive regimen. Material and methods: Renal transplant recipients older than 18 years of age with stable renal function were interviewed from 02/201905/2019. Gastrointestinal symptoms were evaluated using the Gastrointestinal Symptom Rating Scale (GSRS) questionnaire. Higher total scores indicate increased severity in symptoms. Differences in GSRS score were assessed for both total and specific symptom. A $<0.05 p$ was considered statistically significant. Results: 103 patients (male, 63.15\%) were surveyed, average age 36.6 years, average post-transplant time 45.5 months. Ninety-two patients (91\%) had a gastrointestinal symptom. The most common symptoms were hunger pain (65\%), belching (49.5\%) loose stools (42.7\%). Female patients had a higher GSRS score than males $(p<0.05)$. Hemoglobin had a significant inverse correlation to the GSRS score $(p<0.05)$. Individual symptom analysis showed reverse

Citar como: Rivera-Sánchez Y, Martínez-Maldonado M, Reyes-Constantino LR, Domínguez-Rodríguez Á, Márquez-Celedonio FG, Budar-Fernández LF et al. Síntomas gastrointestinales en pacientes receptores de trasplante renal de un centro en México. Rev Mex Traspl. $2021 ; 10$ (2): $64-70$. https://dx.doi.org/10.35366/101413 
correlación inversa significativa a la puntuación GSRS $(\mathrm{p}<$ 0.05). El análisis individual de los síntomas demostró correlaciones inversas de la hemoglobina con náusea, constipación y diarrea. La inmunosupresión (tacrolimus y dosis $>1,000$ $\mathrm{mg}$ /día de micofenolato) tuvo mayor sintomatología gastrointestinal superior $(\mathrm{p}<0.05)$. Conclusiones: La mayoría de los pacientes receptores de trasplante renal sufren molestias gastrointestinales. La introducción de cuestionarios como el GSRS permite al médico obtener información sobre la enfermedad de su paciente y tomar decisiones en su control.

Palabras clave: Trasplante renal, síntomas gastrointestinales, eventos adversos, inmunosupresión.

\section{INTRODUCCIÓN}

El trasplante renal es el procedimiento más efectivo que se utiliza en el tratamiento de pacientes con enfermedad renal. ${ }^{1}$ Si bien el paciente trasplantado no recuperará su calidad de vida previo a la enfermedad renal terminal, existen diferentes beneficios en la calidad de vida del paciente asociados al trasplante renal en comparación con la diálisis. ${ }^{2}$ No obstante, uno de los efectos adversos que se presentan con más frecuencia son los síntomas gastrointestinales ${ }^{1}$ que afectan a $20-40 \%$, los cuales pueden suceder en cualquier segmento del tracto gastrointestinal, mismos que paradójicamente tienden a disminuir su calidad de vida. ${ }^{3}$ Poniendo de lado las enfermedades gastrointestinales preexistentes, las infecciones gastrointestinales y los factores psicosociales, la principal causa de la aparición de síntomas gastrointestinales parece estar asociada a los fármacos utilizados en la terapia de inmunosupresión, especialmente el micofenolato de mofetilo (MMF). ${ }^{4}$ Debido a la posible multicausalidad de la aparición de los síntomas gastrointestinales, resulta difícil diferenciar los casos únicamente asociados al uso de fármacos inmunosupresores de alguna de estas causas.

El manejo de la sintomatología gastrointestinal varía dependiendo de su severidad, la más común es el uso de fármacos protectores gástricos como profilaxis, ${ }^{1}$ aun así, las complicaciones gastrointestinales más severas podrían resultar en una reducción de la dosis de los fármacos inmunosupresores que pueden elevar el riesgo de rechazo del injerto. ${ }^{2}$

El cuestionario Gastrointestinal Symptom Rating Scale (GSRS) se utiliza para evaluar la presencia y severidad de síntomas gastrointestinales. Este cuestionario ha sido validado previamente como instrumento específico en los pacientes de trasplante renal. ${ }^{5} \mathrm{El}$ uso de este cuestionario ha reportado una alta preva- correlations of hemoglobin with nausea, constipation and diarrhea. Immunosuppression (tacrolimus and doses $>1,000$ mg/day of mycophenolate) had greater upper gastrointestinal symptomatology $(p<0.05)$. Conclusions: Most renal transplant recipients experience gastrointestinal symptoms. Introducing questionnaires such as GSRS allows physicians to obtain information about their patient's condition and make decisions in their control.

Keywords: Renal transplantation, gastrointestinal symptoms, adverse events, immunosuppression.

lencia (39\%) de dichos síntomas en poblaciones de trasplante renal. ${ }^{2}$

El objetivo de este trabajo fue determinar la prevalencia de síntomas gastrointestinales en una cohorte de pacientes mexicanos receptores de trasplante renal con función del injerto estable y sin otras enfermedades subyacentes que pudieran causar manifestaciones gastrointestinales. Se evaluó tanto la prevalencia de los síntomas gastrointestinales como las posibles asociaciones a factores del paciente, de su trasplante y de su inmunosupresión.

\section{MATERIAL Y MÉTODOS}

Se realizó un estudio transversal en la consulta externa de trasplante renal de la Unidad Médica de Alta Especialidad Hospital de Especialidades Núm. 14 «Adolfo Ruiz Cortines» de la ciudad de Veracruz, México. Previo consentimiento informado y bajo aprobación del comité local de ética, se encuestó a pacientes incluidos en los siguientes criterios: mayores de 18 años receptores de trasplante renal, con función renal estable (creatinina sérica $<2.5 \mathrm{mg} / \mathrm{dL}$, sin presencia de rechazo activo y sin proceso infeccioso concomitante) y bajo tratamiento inmunosupresor durante el periodo comprendido de febrero a mayo de 2019. Se excluyeron los pacientes que se negaron a realizar el cuestionario.

Se registraron las siguientes variables sociodemográficas y del trasplante: edad, sexo, peso, índice de masa corporal, tensión arterial, tipo de donante (vivo o fallecido), tiempo postrasplante y régimen de inmunosupresión. Asimismo, se registraron los siguientes parámetros de laboratorio: hemoglobina, nitrógeno ureico en sangre, creatinina sérica, depuración de creatinina y glucosa sérica.

Los síntomas gastrointestinales fueron evaluados mediante la aplicación del cuestionario GSRS, el cual 
consiste en 15 preguntas diseñadas para evaluar la severidad de síntomas gastrointestinales superiores e inferiores. ${ }^{4}$ Este instrumento posee cinco subescalas: reflujo, diarrea, constipación, dolor abdominal e indigestión, cada una conformada por tres preguntas. Cada pregunta se evalúa por medio de una puntuación que varía de una (ninguna molestia en absoluto) a siete (molestias muy fuertes). La suma de la puntuación de las 15 preguntas representa la puntuación total, y la suma de la puntuación de las tres preguntas de cada subescala representa la puntuación por subescala. Las puntuaciones (score) totales más altas indican una mayor severidad en los síntomas gastrointestinales. ${ }^{5}$

Análisis estadístico. Las variables continuas y discretas se describen en media, desviación estándar y rango, y las variables categóricas en frecuencias y porcentajes. Los síntomas gastrointestinales de acuerdo a la encuesta GSRS se reportaron en porcentaje. Se realizó correlación de Pearson entre las variables continuas y los síntomas de la escala GSRS. Las diferencias en la puntuación GSRS tanto total como por síntoma específico se analizaron mediante $t$ de Student, $U$ de Mann-Whitney y ANOVA, dependiendo de la variable a estudiar; para este análisis se reagruparon las variables continuas con base en su media estadística. El análisis estadístico se llevó a cabo con el software SPSS versión 25.0 se consideró una $\mathrm{p}<0.05$ como estadísticamente significativa.

\section{RESULTADOS}

Durante el periodo comprendido entre febrero y mayo de 2018 se encuestaron 145 pacientes, de los cuales se excluyeron 14 por falta de apego al tratamiento inmunosupresor, 12 pacientes por tener nivel de creatinina sérica mayor de $2.5 \mathrm{mg} / \mathrm{dL}$, y 16 pacientes por proceso infeccioso gastrointestinal activo al momento de la encuesta; quedando un total de 103 pacientes en su mayoría masculinos (63.15\%), con una edad promedio de $36.6 \pm 12$ años (rango, 18-74). El tiempo promedio postrasplante de los pacientes fue $45.5 \pm 50.3$ meses (rango, 1-216). El resto de sus datos demográficos y clínicos se muestran en la Tabla 1.

De los pacientes, $98.1 \%(n=101)$ ingerían micofenolato de mofetilo, 57 pacientes $(55.3 \%)$ tacrolimus dentro de su régimen de inmunosupresión, 29 (28.2\%) ingerían sirolimus y $13(12.6 \%)$ ciclosporina. De los pacientes, $(91 \%)(n=92)$ utilizaban esteroides. La dosis promedio diaria de micofenolato de mofetilo fue de $1497.5 \pm 511$ mg/día (rango, 0-2,000, mediana 1,500 mg-día).

\section{Síntomas gastrointestinales}

De los pacientes, $92(91 \%)$ tuvieron por lo menos un síntoma gastrointestinal. Los cinco síntomas más comunes fueron dolor de epigástrico urente con ayuno prolongado $(65 \%)$, eructos $(49.5 \%)$, heces sueltas $(42.7 \%)$, tenesmo rectal $(40.8 \%)$ y borborigmos (35.9\%). La Figura 1 indica la severidad de los síntomas gastrointestinales de acuerdo a la encuesta GSRS. La puntuación GSRS promedio de fue $27.3 \pm$ 11 (rango, 15-65). Los pacientes femeninos tuvieron mayor puntuación GSRS $(31.9 \pm 12)$ que los pacientes masculinos $(24.6 \pm 9.4)(p=0.0001$, $U$ de MannWhitney). Los niveles de hemoglobina tuvieron una

Tabla 1: Características clínicas y demográficas.

\begin{tabular}{lc}
\hline & $N=103$ \\
\hline Características & \\
Edad (años) & $36.6 \pm 12.0(18-74)$ \\
Sexo masculino & $63.1 \%(\mathrm{n}=65)$ \\
Peso (kg) & $64.5 \pm 16.3(31.7-11.7)$ \\
Índice de masa corporal (mg/m²) & $24.3 \pm 4.7(13.7-40.4)$ \\
Donador vivo & $78.6 \%(\mathrm{n}=81)$ \\
Etiología & $45.5 \pm 50.3(1-216)$ \\
Tiempo postrasplante (meses) & $12.7 \pm 2.1(7.5-17.2)$ \\
Hemoglobina (g/dL) & $20.4 \pm 7.7(9-46)$ \\
Nitrógeno ureico en sangre (mg/dL) & $1.3 \pm 0.34(0.8-2.2)$ \\
Creatinina sérica (mg/dL) & $66.48 \pm 23.4(30-164)$ \\
Depuración de creatinina $(\mathrm{mL} / \mathrm{min})$ & $93.8 \pm 29.0(65-319)$ \\
Glucosa sérica (mg/dL) & $122.4 \pm 23.0(65-176) / 80.4 \pm 11.9(45-105)$ \\
Presión sistólica y diastólica $(\mathrm{mmHg})$ & \\
\hline
\end{tabular}




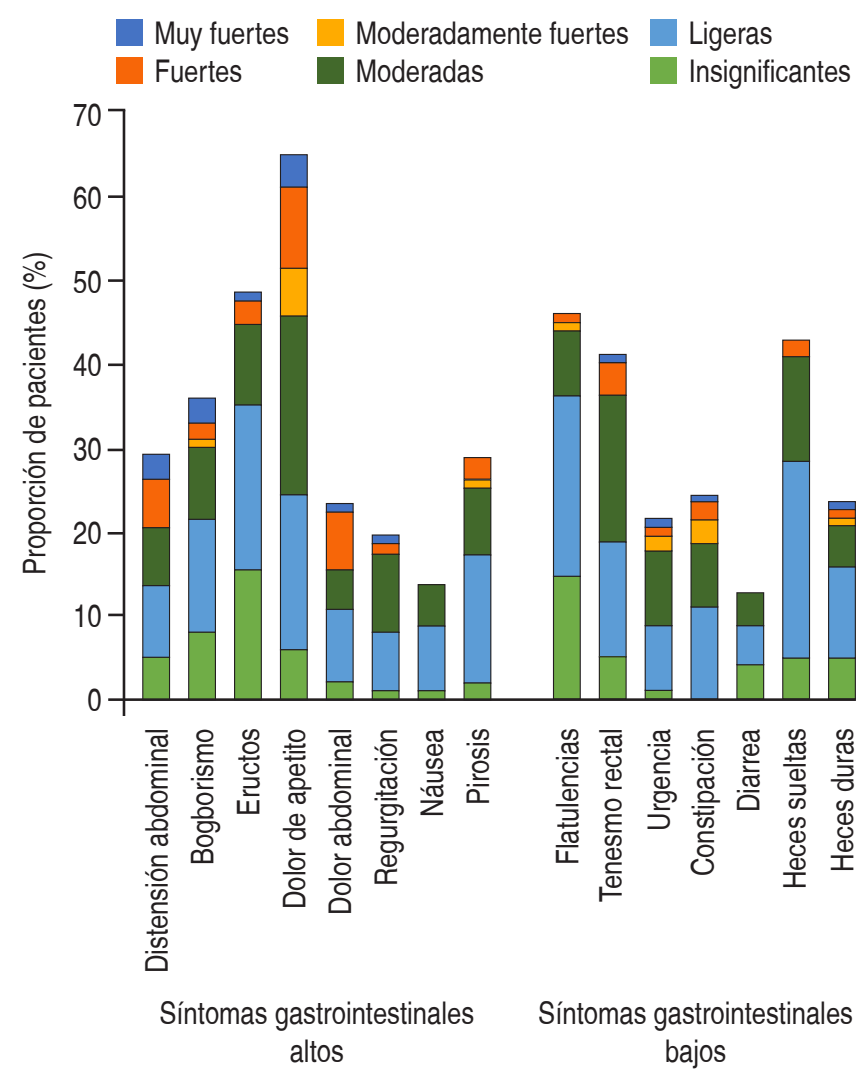

Figura 1: Severidad de síntomas de acuerdo a la escala Gastrointestinal Symptom Rating Scale.

correlación inversa estadísticamente significativa a la puntuación total de GSRS (coeficiente de correlación de Pearson de -0.304, $p=0.007$ ). Los pacientes con hemoglobina $<12.7 \mathrm{~g} / \mathrm{dL}$ tuvieron mayor puntuación total GSRS (31.1 \pm 11.4$)$ que los pacientes con hemoglobina $>12.7 \mathrm{~g} / \mathrm{dL}(23.8 \pm 9.3)(\mathrm{p}=0.003$, t de Student). Los pacientes con régimen de inmunosupresión basado en tacrolimus tuvieron la mayor puntuación total GSRS $(28.9 \pm 10.8)$ que los pacientes con esquema a base de sirolimus $(27.4 \pm 12.9)$ y los pacientes con ciclosporina ( $22 \pm 5.7)$, pero estas diferencias no fueron estadísticamente significativas ( $p=0.1$, ANOVA). No existió correlación ni diferencias significativas entre la dosis total diaria de micofenolato de mofetilo y la puntuación total GSRS.

\section{Análisis individual de síntomas gastrointestinales}

El análisis individual de cada síntoma gastrointestinal reportado en la escala GSRS demostró una correlación inversa estadísticamente significativa de la he- moglobina con náusea, constipación, evacuaciones diarreicas y tenesmo rectal (a menor nivel de hemoglobina mayor sintomatología). La presión diastólica a su vez tuvo una correlación inversa estadísticamente significativa a borborigmo, distensión abdominal y tenesmo rectal (menor presión diastólica mayor sintomatología). El tiempo de trasplante y el nitrógeno ureico también tuvieron correlaciones significativas a otros síntomas (Tabla 2).

El sexo femenino tuvo una puntuación más alta (mayor sintomatología) de tipo gastrointestinal baja (constipación y evacuaciones diarreicas) y distensión abdominal que el sexo masculino, de forma similar que los pacientes con una hemoglobina $<12.7 \mathrm{~g} / \mathrm{dL}$ en síntomas como la constipación y la diarrea $(p<0.05)$. Los pacientes con inmunosupresión (régimen basado en tacrolimus y dosis $>1,000 \mathrm{mg} /$ día de micofenolato de mofetilo) tuvieron mayor sintomatología gastrointestinal superior (distensión y dolor de apetito) que aquellos pacientes con dosis de micofenolato de mofetilo < $1,000 \mathrm{mg} / \mathrm{día}$ y otros medicamentos inmunosupresores. La Tabla 3 muestra los resultados y las diferencias de la puntuación GSRS con diferentes variables estudiadas.

Tabla 2: Correlaciones significativas (Pearson) de puntuación Gastrointestinal Symptom Rating Scale.

\begin{tabular}{|c|c|c|}
\hline Síntoma & Correlación & $p$ \\
\hline \multicolumn{3}{|l|}{ Regurgitación } \\
\hline Nitrógeno ureico & 0.236 & 0.037 \\
\hline \multicolumn{3}{|l|}{ Dolor de apetito } \\
\hline Tiempo postrasplante & -0.336 & 0.001 \\
\hline Dosis de micofenolato & 0.238 & 0.015 \\
\hline \multicolumn{3}{|l|}{ Náusea } \\
\hline Hemoglobina & -0.283 & 0.013 \\
\hline Nitrógeno ureico & 0.225 & 0.047 \\
\hline \multicolumn{3}{|l|}{ Borborigmos } \\
\hline Presión diastólica & -0.198 & 0.023 \\
\hline \multicolumn{3}{|l|}{ Distensión abdominal } \\
\hline Presión diastólica & -0.220 & 0.034 \\
\hline \multicolumn{3}{|l|}{ Constipación } \\
\hline Peso & -0.205 & 0.045 \\
\hline Hemoglobina & -0.260 & 0.022 \\
\hline \multicolumn{3}{|l|}{ Diarrea } \\
\hline Hemoglobina & -0.249 & 0.029 \\
\hline \multicolumn{3}{|l|}{ Heces duras } \\
\hline Glucosa & 0.305 & 0.007 \\
\hline \multicolumn{3}{|l|}{ Tenesmo rectal } \\
\hline Tiempo postrasplante & -0.198 & 0.045 \\
\hline Hemoglobina & -0.229 & 0.045 \\
\hline Presión diastólica & -0.220 & 0.034 \\
\hline
\end{tabular}


Tabla 3: Puntuación Gastrointestinal Symptom Rating Scale significativo de acuerdo a variables categorizadas.

\begin{tabular}{|c|c|c|}
\hline Síntoma & Puntuación & $p$ \\
\hline \multicolumn{3}{|l|}{ Dolor de apetito } \\
\hline \multicolumn{3}{|c|}{ Tiempo postrasplante (meses) } \\
\hline$<27$ & $3.5 \pm 1.80$ & \\
\hline$>27$ & $2.49 \pm 1.60$ & $0.004^{*}$ \\
\hline \multicolumn{3}{|c|}{ Régimen de inmunosupresión } \\
\hline Tacrolimus & $3.5 \pm 1.80$ & \\
\hline Sirolimus & $2.6 \pm 1.60$ & \\
\hline Ciclosporina & $1.9 \pm 1.25$ & $0.006^{\ddagger}$ \\
\hline \multicolumn{3}{|c|}{ Dosis de micofenolato (mg/día) } \\
\hline$<1,000$ & $2.54 \pm 1.70$ & \\
\hline$>1,000$ & $3.29 \pm 1.80$ & $0.038^{\S}$ \\
\hline \multicolumn{3}{|l|}{ Borborigmos } \\
\hline \multicolumn{3}{|l|}{ Presión diastólica (mmHg) } \\
\hline$<80$ & $2.2 \pm 1.70$ & \\
\hline$>80$ & $1.5 \pm 0.90$ & 0.061 \\
\hline \multicolumn{3}{|l|}{ Distensión abdominal } \\
\hline \multicolumn{3}{|l|}{ Sexo } \\
\hline Femenino & $2.6 \pm 2.00$ & \\
\hline Masculino & $1.4 \pm 1.00$ & $0.002^{*}$ \\
\hline \multicolumn{3}{|c|}{ Régimen de inmunosupresión } \\
\hline Tacrolimus & $2.26 \pm 1.80$ & \\
\hline Sirolimus & $1.55 \pm 1.40$ & \\
\hline Ciclosporina & $1.08 \pm 0.20$ & $0.027^{\ddagger}$ \\
\hline \multicolumn{3}{|l|}{ Constipación } \\
\hline \multicolumn{3}{|l|}{ Peso (kg) } \\
\hline$<64.5$ & $2 \pm 1.50$ & \\
\hline$>64.5$ & $1.2 \pm 1.00$ & $0.007^{\S}$ \\
\hline \multicolumn{3}{|l|}{ Hemoglobina (mg/dL) } \\
\hline$<12.7$ & $2.35 \pm 1.75$ & \\
\hline$>12.7$ & $1.38 \pm 1.07$ & $0.005^{\S}$ \\
\hline \multicolumn{3}{|l|}{ Sexo } \\
\hline Femenino & $2.3 \pm 1.70$ & \\
\hline Masculino & $1.3 \pm 0.90$ & $0.001^{*}$ \\
\hline \multicolumn{3}{|l|}{ Diarrea } \\
\hline \multicolumn{3}{|l|}{ Hemoglobina (mg/dL) } \\
\hline$<12.7$ & $1.46 \pm 0.90$ & \\
\hline$>12.7$ & $1.13 \pm 0.50$ & $0.006^{\S}$ \\
\hline \multicolumn{3}{|l|}{ Sexo } \\
\hline Femenino & $1.45 \pm 0.90$ & \\
\hline Masculino & $1.14 \pm 0.52$ & $0.045^{\star}$ \\
\hline
\end{tabular}

${ }^{*} \mathrm{t}$ de Student, ${ }^{\ddagger}$ ANOVA, ${ }^{\S}$ Mann-Whitney.

\section{DISCUSIÓN}

Los resultados de nuestro estudio demuestran una elevada frecuencia de síntomas gastrointestinales en pacientes receptores de trasplante renal. Asimismo, estos síntomas (que pueden ser tanto gastrointestinales altos como gastrointestinales bajos) se presentaron con más frecuencia en pacientes de sexo feme- nino, pacientes con niveles séricos de hemoglobina menores y pacientes con menos tiempo de trasplante. La inmunosupresión basada en tacrolimus y el uso de micofenolato se asociaron a la presencia de síntomas gastrointestinales generales y de algunos de ellos en forma específica.

Los síntomas gastrointestinales en pacientes receptores de trasplante tienen un amplio rango de presentación, pueden ir desde $11 \%{ }^{6}$ hasta cifras alrededor de $90 \% .^{2-4}$ No obstante este rango, se reconoce que la población receptora de trasplante renal presenta con más frecuencia estos síntomas que la población en general. ${ }^{4}$

En nuestro caso, la prevalencia de síntomas gastrointestinales en México fue recientemente analizada (2014) por medio del estudio SIGAME, ${ }^{7}$ en el que podemos encontrar que la sintomatología gastrointestinal en la población mexicana puede llegar a tener síntomas específicos hasta $50-60 \%$ de los casos (agruras, regurgitación y síntomas dispépticos), y que a pesar de que esta prevalencia es relativamente alta, es inferior a $90 \%$ que se observa en pacientes receptores de trasplante renal y que, a diferencia de la población receptora de trasplante, los síntomas en el estudio SIGAME son más frecuentes en varones que en mujeres. ${ }^{7}$ Es interesante destacar que la población encuestada en nuestro estudio tiene características sociodemográficas diferentes a otras encuestas previamente publicadas en receptores de trasplante renal. Nuestros pacientes tienen un promedio de edad más joven (36 años) y menos tiempo de trasplante (3.7 años) en comparación con otros cuya edad es cercana a 50 años y el tiempo promedio de trasplante es superior a cinco años. ${ }^{1-3}$

La evaluación específica de los síntomas gastrointestinales en nuestro estudio nos dice que el dolor epigástrico urente con ayuno prolongado y los eructos fueron los más frecuentes en cifras cercanas y superiores a $50 \%$. Cuando se evalúan los síntomas de forma particular, éstos son variados dependiendo la publicación: diarrea, ${ }^{1,3}$ flatulencia, ${ }^{2}$ dolor ${ }^{4}$ y náusea. ${ }^{6} \mathrm{~A}$ pesar de estas diferencias, lo que es constante en los estudios es la asociación del uso de tacrolimus y del micofenolato de mofetilo con la presencia de síntomas gastrointestinales. ${ }^{1-4}$ En el caso del micofenolato de mofetilo, los eventos adversos gastrointestinales son ampliamente conocidos, ya que afectan hasta $45 \%$ de los pacientes. ${ }^{8,9}$ Estos síntomas/eventos pueden llevar a la manipulación de la dosis, en particular reducción de la misma, la cual además de generar tolerabilidad del fármaco puede aumentar el riesgo de algún episodio 
de rechazo agudo del injerto y afectar negativamente la sobrevida del injerto..$^{10,11} \mathrm{La}$ asociación de tacrolimus con diarrea/constipación también se ha descrito con anterioridad. ${ }^{12}$ Los niveles de micofenolato de mofetilo y la dosis superior a $1,000 \mathrm{mg} /$ día en nuestro estudio tuvieron una correlación directamente proporcional al dolor de apetito y en el caso del tacrolimus, aunque no tuvieron significancia estadística, presentaron mayor puntuación global del GSRS, también se relacionaron significativamente a la distensión y al dolor. La presencia de síntomas gastrointestinales asociados al sexo femenino en la población receptora de trasplante renal ya había sido previamente descrita ${ }^{3}$ así como la asociación a los niveles bajos de hemoglobina. ${ }^{1}$ El micofenolato de mofetilo y la azatriopina pueden ser mielotóxicos debido a su efecto antiproliferativo y producen supresión de la médula ósea, ${ }^{13}$ son dosis dependientes y pueden inducir a anemia. El estudio TRESAM resaltó en los resultados de su encuesta una fuerte correlación entre los niveles de hemoglobina y la función del injerto renal, en el cual la mayoría de los pacientes anémicos tenían niveles de creatinina sérica $>2 \mathrm{mg} / \mathrm{dL} .{ }^{14}$ Es difícil poder diferenciar si la anemia es el detonante de los síntomas gastrointestinales, o si los fármacos inmunosupresores que provocan anemia también están generando la sintomatología gastrointestinal. No encontramos evidencia que sugiera el mecanismo de la anemia con los síntomas gastrointestinales, por lo que se podría pensar en una asociación entre los síntomas, presencia de anemia y los fármacos inmunosupresores, y al no estar documentado se podría estudiar en el futuro.

No existen grandes antecedentes sobre la presencia de síntomas gastrointestinales en pacientes receptores de trasplante renal en nuestro país. Sólo un estudio en pacientes receptores de trasplante renal pediátrico que evaluaba la conversión de micofenolato de mofetilo a micofenolato de capa entérica, reveló una disminución de la presencia y severidad de síntomas gastrointestinales en dicha población. ${ }^{15}$ No obstante, éste no era su objetivo principal, por lo que podemos decir que nuestra investigación es el primer estudio mexicano sobre frecuencia y presencia de síntomas gastrointestinales en población trasplantada de México. Es importante resaltar el impacto negativo que tiene esta sintomatología en la calidad de vida del paciente debido a la presencia de modificaciones corporales y al abordaje multidisciplinario de las mismas. ${ }^{16}$ También se observa repercusión económica para el paciente y el proveedor de salud, ya que al redu- cir la dosis del fármaco inmunosupresor con la finalidad de disminuir la sintomatología hay mayor riesgo de presentar rechazo agudo del injerto y esto repercute en erogaciones superiores de dinero en el cuidado del paciente al tratar diferentes tipos y episodios de rechazo. ${ }^{17}$

\section{CONCLUSIONES}

La mayoría de los pacientes receptores de trasplante renal sufren molestias gastrointestinales. Se resalta la importancia de un adecuado diagnóstico y manejo de las mismas para optimizar el régimen de inmunosupresión de los pacientes, calidad de vida y menor riesgo de falla al tratamiento. La introducción de estos cuestionarios como el GSRS permite al médico tratante obtener información más detallada acerca de la enfermedad de su paciente para tomar decisiones adecuadas en su control.

\section{REFERENCIAS}

1. Gil-Vernet S, Amado A, Ortega F et al. Gastrointestinal complications in renal transplant recipients: MITOS study. Transplant Proc. 2007; 39: 2190-2193.

2. Ponticelli C, Colombo D, Novara $M$ et al. Gastrointestinal symptoms impair quality of life in Italian renal transplant recipients but are under-recognized by physicians. Transpl Int. 2010; 23: 1126-1134.

3. Savvidaki E, Papachristou E, Kazakopoulos $P$ et al. Gastrointestinal disorders after renal transplantation. Transplant Proc. 2014; 46: 3183-3186.

4. Ekberg $\mathrm{H}$, Kyllonen L, Madsen $\mathrm{S}$ et al. Increased prevalence of gastrointestinal symptoms associated with impaired quality of life in renal transplant recipients. Transplantation. 2007; 83: 282289.

5. Kleinman L, Kilburg A, Machnicki G et al. Using Gl-specific patient outcome measures in renal transplant patients: validation of the GSRS and GIQLI. Qual Life Res. 2006; 15: 1223-1232.

6. Nagaraj N, Kahan B, Adler DG. Gastrointestinal complications in renal transplant patients: a large, single-center experience. Dig Dis Sci. 2007; 52: 3394-3395.

7. Remes-Troche JM. Síntomas gastrointestinales en México. Un estudio epidemiológico. 2015. Editorial ASECOM, México D.F. México. ISBN 978-607-96924-0-7.

8. Sollinger HW, for the US Renal Transplant Mycophenolate Mofetil Study Group. Mycophenolate mofetil for the prevention of acute rejection in primary cadaveric renal allograft recipients. Transplantation 1995; 60: 225-232.

9. The Tricontinental Mycophenolate mofetil Renal Transplantation Study Group. A blinded, randomized clinical trial of mycophenolate mofetil for the prevention of acute rejection in cadaveric renal transplantation. Transplantation. 1996; 61: 1029-1037.

10. Tierce JC, Porterfield-Baxa J, Petrilla A et al. Impact of mycophenolate mofetil (MMF)-related gastrointestinal complications and MMF dose alterations on transplant outcomes and healthcare costs in renal recipients. Clin Transplant. 2005; 19: $779-784$. 
11. Laftavi MR, Hai F, Feng Let al. Mycophenolic acid dose reductions result in poor long-term renal allograft survival: Comparison between mycophenolate sodium and mycophenolate mofetil. Transplant Proc. 2011; 43: 478-481.

12. Pirsch JD, Miller J, Deierhoi MH et al. A comparison of FK506 and CsA for immunosuppression after cadaveric kidney transplantation. Transplantation. 1997; 63: 977-983.

13. Martin-Valencia A, Perello-Carrascosa M, Seron-Micas D. Anemia en paciente trasplantado renal secundaria a infección por parvovirus B19. Nefrologia Sup Ext. 2012; 3 (5): 22-26.

14. Vanrenterghem Y, Ponticelli C, Morales JM. Prevalence and management of anemia in renal transplant recipients: a European survey. Am J Transplant. 2003; 3: 835-845. ISSN 1600-6135.

15. Reyes $\mathrm{H}$, Hernandez AM, Valverse $\mathrm{S}$ et al. Efficacy and safety of conversion of mycophenolate mofetil to enteric-coated mycophenolate sodium in Mexican renal transplant children. Ped Transp. 2010; 14: 746-752.

16. Pérez BC, Moyano EMC, Estepa AM, Crespo MR. Factores asociados a calidad de vida relacionada con la salud de pacientes trasplantados de riñón. Enferm Nefrol. 2015; 18 (3): 204-226.

17. Martinez-Mier G, Salazar-Ramirez A. The cost of gastrointestinal adverse events and the impact of dose-reductions/ discontinuations on acute rejection in kidney transplant patients of mycophenolate mofetil-related compared to enteric-coated Mycophenolate sodium: a pharmacoeconomic study. Transplant Proc. 2016; 48 (2): 588-595.

\author{
Correspondencia: \\ Dr. Gustavo Martínez-Mier \\ Corporativo San Gabriel \\ Calle Alacio Pérez Núm. 918-314, \\ Col. Ignacio Zaragoza, 91910 , \\ Veracruz, Ver. México, \\ E-mail: gmtzmier@gmail.com \\ gmtzmier@hotmail.com
}

